\title{
"Los caminos no tienen dexo ni género". Una propuesta para la incorporación de mujeres en el espacio público comunitario en el marco de la infraestructura vial cantonal, Naranjo, Costa Rica
}

"The roads are sexless and genderless" A proposal for the incorporation of women in Communitary Public Space under the framework of the cantonal road infrastructure, Naranjo, Costa Rica

"As estradas são sem sexo e sem género". Proposta para a incorporação da mulher no espaço público comunitário no âmbito da infra-estrutura rodoviária cantonal, Naranjo, Costa Rica

Yolanda Pérez-Carrillo Académica e investigadora Escuela de Sociología Universidad Nacional Costa Rica

Recibido: 11/01/2020 - Aceptado: 15/04/2020

Resumen

El presente artículo presenta una propuesta de proyecto cuyo fin buscó definir, de forma operativa, una estrategia pertinente para que, desde el ámbito del gobierno local municipal, se definan acciones para que

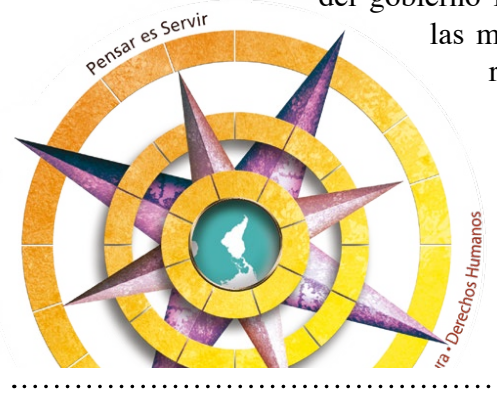

ren en procesos de formación y capacitación que les permitan dinámicas organizativas comunitarias, en primera instancia, $\mathrm{y}$, posteriormente, acceder a las estructuras políticas municipales como enlaces 
comunales-municipales, de tal forma que tengan incidencia en las decisiones locales. Se retoman cinco ejes de formación y capacitación sobre los cuales se debe fundamentar la incorporación de las mujeres en las cuestiones relacionadas al camino, sobre todo los rurales, donde las mujeres han sido invisibilizadas.

Palabras claves: Desigualdad, vialidad, comunidad, municipio, género, infraestructura vial en Costa Rica

\begin{abstract}
This article presents a project proposal that seeks to operationally define a relevant strategy for the local municipal government to define actions so that women from nearby localities are incorporated into education and training processes that will allow them to join community organizational dynamics, in the first instance, and later on to municipal political structures as community-municipal links, in such a way that they can have an influence on local decisions. Five areas of education and training are taken up in order to provide a basis for the incorporation of women in issues related to the road, especially in rural areas, where women have been made invisible.
\end{abstract}

Keywords: inequality, roads, community, municipality, gender, road infrastructure in Costa Rica

Resumo

Este artigo apresenta uma proposta de projecto cujo objectivo era definir de forma operativa uma estratégia relevante para que, a partir do nível do governo local municipal, sejam definidas acções para que as mulheres das localidades próximas sejam incorporadas em processos de formação e de capacitação que lhes permitam integrar as dinâmicas organizacionais da comunidade, em primeiro lugar, e posteriormente nas estruturas políticas municipais como elos comunidade-municipal, de modo a que tenham impacto nas decisões locais. São retomadas cinco áreas de educação e formação, nas quais a incorporação das mulheres nas questões relacionadas com as estradas deve basear-se, especialmente nas zonas rurais onde as mulheres foram tornadas invisíveis.

Palavras chave: desigualdade, estradas, comunidade, município, género, infra-estruturas rodoviárias na Costa Rica

En Costa Rica, al igual que en diversos países latinoamericanos, el espacio público ha sido asignado, por un sistema patriarcal, a los hombres, y el espacio privado, a las mujeres, sumado a esto, hay campos de acción que, por estigmatización, son asignados a los hombres, tal es el caso de lo relacionado con el tema de la infraestructura vial. Se establece una conexión con el trabajo pesado, el cual, por la fuerza física, se les endosa a los hombres, con lo que se anula, por completo, que más allá de

110 "Los caminos no tienen sexo ni género"

Una propuesta para la incorporación de mujeres en el espacio público comunitario en

el marco de la infraestructura vial cantonal, Naranjo, Costa Rica

Yolanda Pérez-Carrillo 
la ejecución de las obras físicas, se requiere un proceso de gestión política, coordinación, planificación, organización , ejecución de obra, manejo político, formación, capacitación, las cuales pueden ser asumidas por mujeres de las comunidades involucradas.

Por ello, desde esta propuesta, se plantean posibles escenarios de incorporación de las mujeres en este campo. Con ello se busca superar la desigualdad en el espacio público, ya que las mujeres son relegadas al espacio privado, al cuido de los niños y niñas, además de a las labores del hogar, y se invisibiliza el aporte que puedan hacer en las organizaciones locales comunitarias.

Con la creación de la Ley 8114 de Simplificación y Eficiencia Tributaria y el Reglamento 34624 en el año 2001, se crean figuras organizativas municipales y se marca un proceso de descentralización vial en el que se transfieren recursos a los gobiernos locales, y se reafirman las competencias, que ya están contempladas en el Código Municipal, en el cual se establece que la red vial cantonal es competencia municipal.

Entre las diversas estructuras locales se encuentra la Junta Vial Cantonal (JVC), órgano colegiado que marca el rumbo de la ejecución de los recursos asignados, además de la Unidad Técnica de Gestión Vial Municipal (UTGVM), encargada de dar asistencia técnica y social a las comunidades, establecer coordinación entre las instituciones, además asesorar a la JVC, entre otras actividades. En dicha legislación, precisamente se señala:

“Artículo 8.- Modalidad participativa de ejecución de obras la que refiere a:

La ejecución de los recursos provenientes de la Ley No. 8114 se realizará bajo la modalidad participativa de ejecución de obras. Dicha modalidad se refiere a la coordinación y cooperación que se establece entre la municipalidad, el Gobierno Central, las organizaciones comunales y la sociedad civil de un cantón, con la finalidad de planificar, ejecutar, controlar y evaluar obras de diversa índole, contempladas dentro de la conservación y construcción vial, en el entendido que la ejecución de recursos no implica el traslado horizontal de los mismos de una organización a otra.

Además de esta legislación, se retoma la Ley Especial para la Transferencia de Competencias: Atención Plena y Exclusiva de la Red Vial Cantonal 'N. 9329, que también alude a la modalidad participativa de ejecución de obras. 
Ahora bien, la modalidad participativa de obras refiere al trabajo colaborativo entre instituciones, organizaciones locales, también denominada Conservación Vial Participativa, es "una alternativa de trabajo conjunto y coordinado, que incorpora todos los elementos de planificación, organización y ejecución efectiva de obras viales en lastre y tierra, con un fuerte énfasis en los aspectos sociales de participación, solidaridad, equidad y respeto mutuo" (MOPT/ GTZ, 2001,p. 5).

En esta modalidad participativa es donde se inicia la apertura para las comunidades $\mathrm{u}$ otras organizaciones locales; sin embargo, la modalidad participativa no significa que se incorporen hombres y mujeres en ese espacio público, ya que la participación de las mujeres ha sido vetada por la estigmatización de roles donde se les asignan las labores del hogar a estas, así se limita su incorporación en los espacios públicos y se genera, con ello, desigualdad de participación comunitaria, política, y de incidencia local, ya que al no ser partícipes de estos espacios no pueden decidir, y menos incidir en cuestiones de caminos, con la limitante de que se omite que ellas son usuarias directas día tras día, en el tanto son las que trasladan a menores de edad a los centros educativos, centros hospitalarios, entre otras actividades más.

Por ello, desde el vector de la desigualdad, se hace necesaria una mejor compresión del espacio público, como este espacio de dinámicas locales, políticas, económicas y culturales, y del que las mujeres deben ser parte activa y no pasiva.

En este sentido, la construcción de espacios públicos es esencial. Jürgen Habermas plantea que "la esfera o espacio de la opinión pública es, ciertamente, un fenómeno social tan elemental como la acción, el actor, el grupo o el colectivo es una red para la comunicación de contenidos y tomas de postura, es decir, de opiniones" (Habermas, 1998, p. 440).

Es decir, se requiere un espacio público que genere las condiciones para que las mujeres se incorporen, de forma activa, en las decisiones locales, de manera coordinada con los hombres, quienes tradicionalmente han estado dirigiendo, organizado y decidiendo en ese campo masculinizado, y que socialmente les asigna roles a hombres y mujeres. Se le asigna, a las mujeres, el ámbito privado del hogar y se relegan de lo público, de tal forma, los hombres permanecen vinculados al trabajo remunerado, y las mujeres continúan

112 "Los caminos no tienen sexo ni género"

Una propuesta para la incorporación de mujeres en el espacio público comunitario en

el marco de la infraestructura vial cantonal, Naranjo, Costa Rica

Yolanda Pérez-Carrillo 
pensadas como responsables únicas y exclusivas del trabajo doméstico, así como del cuido: Existe una anulación e invisibilidad del accionar de las mujeres en los caminos rurales, por ello, desde un procesos de extensión universitaria, se debe operativizar de manera lógica y articulada el empoderamiento de estas mujeres mediante procesos de formación y capacitación, aunado a su incorporación en las organizaciones vinculadas al quehacer de los municipios.

A partir de lo expresado líneas atrás, se considera oportuno que las mujeres sean parte de diversos procesos, que les permitan incidir en estos espacios públicos vetados y que, al final, las dejan en estado de desigualdad organizativa y comunal, ya que se les priva de ser actoras directas de transformación local.

En consecuencia, con lo descrito líneas atrás, entonces, se hace necesario y pertinente que se desarrollen las siguientes acciones concretas:

1. Promover desde el Estado la igualdad, con base en los objetivos de la Agenda 2030.

2. Marcar procesos de descentralización, fortaleciendo los gobiernos locales, como espacios más concretos donde se pueden operativizar algunos objetivos.
3. En el marco del tema infraestructura vial cantonal, incorporar, de una forma más activa, a las mujeres en los espacios de decisión cantonal (Junta Vial Cantonal), ya que al ser las que lideran los diversos procesos, deben entonces decidir e incidir.

4. Desde las Unidades Técnicas y de Gestión Vial (oficinas municipales) orientar procesos de formación, capacitación y organización, de tal forma que permitan a las mujeres empoderarse y ser parte de los procesos políticos comunitarios y locales (para que las mujeres asuman puestos políticos en las alcaldías, primero hay que fortalecer su accionar comunitario, aunado a la ampliación de una mirada más macro.

5. Articular a los actores locales (sociedad civil) e institucionales que posibiliten los procesos locales.

6. Incluir, a las universidades públicas, en procesos de investigación y extensión, para promover que las mujeres asuman mayor participación en espacios públicos en los que logren incidir.

7. Articular esfuerzos municipales que trabajen temas de género (oficina de la mujer, oficina

"Los caminos no tienen sexo ni género" 113 
ambiental, oficina de gestión vial, oficina de proyección social, entre otras), de tal forma que se hagan propuestas conjuntas y articuladas, y se establezcan como políticas de incidencia locales.

8. Monitorear y dar seguimiento a las diversas acciones.

9. Divulgar las experiencias locales que sirvan como insumo para aplicar réplicas en otros municipios del país.

Si bien hay acciones concretas que permitan una incidencia de las mujeres en los espacios públicos, es necesario, primero, que exista voluntad política de los gobiernos locales, que son los entes más cercanos a las comunidades.

Cabe señalar que, además de lo registrado anteriormente, se requiere impulsar procesos de formación y capacitación, de tal forma que se potencien las capacidades de las mujeres, esto porque, en la mayoría de los casos, tienen el conocimiento para incorporarse, pero la inseguridad de ser parte de diversos procesos les impide una incorporación activa.
Entre los procesos de formación y capacitación que se considera oportuno incorporar se encuentran los siguientes ejes temáticos: organización, legislación vial, mantenimiento manual de caminos, gestión de recursos y elaboración de presupuestos, coordinación institucional. Si bien puede haber otras temáticas, se considera que estas deben ser las prioritarias para una propuesta de integración que permita y facilite que las mujeres se incorporen, de forma activa, en los espacios públicos. Es por ello que, desde cada perspectiva, se fundamentan algunos aspectos orientadores.

\section{Organización}

1. La organización es la base principal de toda acción.

2. Es fundamental que las mujeres conozcan qué hace una organización de comité de caminos, además de que deben conocer las funciones. correspondientes según puesto, eso contribuye a que se organicen de mejor forma.

3. Si las mujeres se organizan en la comunidad, pueden sacar cualquier iniciativa adelante, es decir, ejecutar diversos proyectos, $\mathrm{y}$ de esta forma obtener mejor beneficio. 
4. Las organizaciones, si funcionan bien, pueden generar mayores espacios de incorporación de otras mujeres de las comunidades.

5. La capacitación en organización orienta diversos procesos comunitarios, además de ser articuladora con otras organizaciones e instituciones.

6. Asegura que los procesos sean inclusivos y participativos.

7. Todo debe tener un orden para poder trabajar productiva y eficazmente, logrando alcanzar objetivos y metas, además de una buena administración en recursos.

\section{Legislación vial}

1. Las mujeres deben conocer las leyes que rigen el sistema de caminos, además de los derechos y deberes de las personas de la comunidad.

2. Con un conocimiento oportuno, pueden contribuir al desarrollo comunitario y actuar conforme a la legislación.

3. El conocimiento de estas les permitirá tener un equilibrio en el ser y el actuar.
4. Reconocer que los caminos son responsabilidad de hombres y mujeres implica que las mujeres puedan participar de la toma de decisiones de forma oportuna y coordinada.

\section{Mantenimiento manual de caminos}

1. Las mujeres deben incorporarse de forma conjunta con los hombres en la protección de los caminos y minimizar el deterioro.

2. Aplicar los conocimientos y leyes en el mantenimiento de los caminos, mediante notificaciones de las organizaciones y de la coordinación municipal.

3. Realizar diagnósticos de necesidades del camino de forma conjunta con los hombres, jóvenes, personas menores.

4. Coordinación y motivación constante con cada dueño o dueña de finca, a fin de que realice lo que le corresponde, según lo establecido en la legislación nacional y local.

5. El punto anterior está condicionado a lo que se establece respecto a las competencias de cada vecino o vecina, y lo que debe hacerse de forma organizada.

"Los caminos no tienen sexo ni género" 115 


\section{Gestión de recursos y ela- boración de presupuestos}

1. Las mujeres, de forma conjunta con los hombres, deben incorporarse en los procesos de gestión de recursos para la ejecución de obras en los caminos.

2. La administración de los recursos debe hacerse de forma equilibrada entre mujeres y hombres, según los roles asignados a lo interno del grupo comunitario.

3. Gestionar recursos con cooperativas, otras organizaciones e instituciones recursos para una inversión oportuna, y según las necesidades locales.

4. Orientación oportuna de cómo realizar la gestión de los recursos para proyectos, es decir, lograr identificar las posibles fuentes de financiamiento, así como las modalidades y los procesos a cumplir para su obtención.

5. Se deben orientar estrategias de negociación, es decir, saber pedir lo que se necesita.

6. Además, en la gestión de recursos, deben direccionarse los recursos que se adquieren según una planificación previa.
7. Con respecto a los presupuestos, es necesario vigilar que los recursos se ejecuten, a partir de estudios técnicos, económicos y ambientales.

8. Los trabajos deben ser enfocados de acuerdo con las posibilidades presupuestarias.

9. Resulta fundamental que las mujeres tengan conocimiento de cómo elaborar los presupuestos.

\section{Coordinación} institucional

1. Se requiere que las mujeres conozcan cuáles son las acciones básicas para una adecuada coordinación institucional, las personas enlaces, entre otras.

2. Disponer de condiciones que les permitan realizar gestión política con representantes institucionales.

3. Realizar ejecución conjunta con las instituciones según sus competencias y responsabilidades.

4. Articular esfuerzos con diversas instituciones para optimizar los recursos existentes.

5. Disponer de información fluida que facilite la comunicación 
acertada entre instituciones, ONG, mujeres y hombres de la comunidad.

6. Disponer de los procedimientos a seguir para cada institución, a fin de realizar gestiones oportunas según sus competencias.

A partir de los ejes considerados en la propuesta, se espera iniciar con un proceso comedido que permita cambiar paradigmas establecidos socialmente en las comunidades rurales, de tal forma que se deconstruya lo establecido en el que las mujeres asumen el hogar y los hombres el espacio público, así pues, las mujeres deben incorporarse en ese espacio público comunitario, donde también sufren violencia política, en el tanto se les anula su participación en puestos de decisión local. Es necesario romper con la simbolización cultural que acuña Marta Lamas (1996) y en el que refiere el género como simbolización

... esa simbolización cultural construida a partir de la diferencia sexual, rige el orden humano y se manifiesta en la vida social, política y económica. Entender qué es y cómo opera el género nos ayuda a vislumbrar cómo el orden cultural produce percepciones específicas sobre las mujeres y los hombres, percepciones que se erigen en prescripciones sociales con las cuales se intenta normar la convivencia. (p. 1)

Convivencia que debe ser basada en el respeto y en el buen vivir, que permita y genere las condiciones para que las mujeres asuman nuevos roles en el desarrollo comunitario, los cuales han sido establecidos socialmente, y que pueden ser modificados de la misma forma.

Sin embargo, desde mi perspectiva, considero que las mujeres, para ser parte de un proceso de incorporación en cualquier temática de trabajo estigmatizada para los hombres, primero deben ser parte de procesos de formación y capacitación, de tal forma que se les oriente en el accionar concreto. Son estos procesos de transferencia de conocimiento, inclusive de otras mujeres, lo que puede contribuir de manera positiva para una incorporación activa y oportuna.

La categoría de género, dependiendo del contexto en que se utiliza, hará alusión a lo masculino (fuerte), y a lo femenino (débil). En el tema que nos compete, referido a los caminos, se ha creído que estos son sinónimos de trabajo pesado, y solo pueden ser realizados por los hombres, anulando por completo que, detrás de la construcción de estos, hay diversas acciones necesarias para poder ver

"Los caminos no tienen sexo ni género" 117 
las obras terminadas, además del monitoreo, seguimiento, coordinación, gestión de recursos, organización, entre otras actividades que pueden ser desarrolladas por hombres y mujeres en igualdad de condiciones, es decir, superando esas desigualdades instauradas en las comunidades locales, en la que se subestima el aporte que pueden hacer las mujeres en campos tan masculinizados como los caminos, en este caso los rurales.

El género produce un imaginario social con una eficacia simbólica contundente y, al dar lugar a concepciones sociales y culturales sobre la masculinidad y feminidad, es usado para justificar la discriminación por sexo (sexismo) y por prácticas sexuales (homofobia). (Lamas, 2000, p. 4)

El sistema social es el que da soporte para las discriminación, es aquí donde los hombres y mujeres, en procesos de socialización primaria y secundaria, en la mayoría de los casos, se siguen permeando nuevas generaciones en las que las mujeres son parte de un sistema desigual, y en el que se subestima su capacidad $\mathrm{y}$, por ende, se les impide el acceso a lo público, pero sobre todo a incorporarse a espacios estigmatizadas asumidos, tradicionalmente, por los varones.
$\mathrm{Al}$ sostenimiento del orden simbólico contribuyen hombres y mujeres, reproduciéndose y reproduciéndolo. Los papeles cambian según el lugar o el momento; pero mujeres y hombres, por igual, son los soportes de un sistema de reglamentaciones, prohibiciones y opresiones recíprocas (Lamas, 2000).

Por su parte, Bourdieu (2000) plantea dos categorías significativas en torno a la composición del género, las cuales son el orden social y la división sexual del trabajo.

[El orden social] funciona como una inmensa máquina simbólica que tiende a ratificar la dominación masculina en la que se apoya: es la división sexual del trabajo, distribución muy estricta de las actividades asignadas a cada uno de los dos sexos, de su espacio, su momento, sus instrumentos; es la estructura del espacio, con la oposición entre el lugar de reunión o el mercado, reservados a los hombres, y la casa, reservada a las mujeres, o, en el interior de ésta, entre la parte masculina, como del hogar, y la parte femenina, como el establo, el agua y los vegetales; es la estructura del tiempo, jornada, año agrario, o ciclo de vida, con los momentos de ruptura, masculinos, y los largos periodos de gestación, femeninos. (Bourdieu, 2000, p. 22) 


\section{Y la división sexual del trabajo, se entiende como:}

La diferencia biológica entre los sexos, es decir, entre los cuerpos masculino y femenino, y, muy especialmente, la diferencia anatómica entre los órganos sexuales, puede aparecer de ese modo como la justificación natural de la diferencia socialmente establecida entre los sexos, y en especial de la división sexual del trabajo. (El cuerpo y sus movimientos, matrices de universales que están sometidas a un trabajo de construcción social, no están ni completamente determinados en su significación, sexual especialmente, ni completamente indeterminados, de manera que el simbolismo que se les atribuye es a la vez convencional y «motivado», percibido por tanto como casi natural. (Bourdieu, 2000, p. 24)

Así pues, este autor asume estas categorías a partir de las responsabilidades, asignaciones, recompensas $\mathrm{u}$ otros elementos que hacen parte de las tareas, conductas y prácticas que las personas, según su sexo biológico (justificador natural), "deben" realizar. De este modo, más allá de comprender cómo se da su mundo y relaciones en las organizaciones viales, se pueden comprender sus actividades a raíz de un contexto y una visión ya establecida por la dominación masculina de la cual habla Bourdieu, y que se manifiesta en las dinámicas alrededor de los caminos rurales.

\section{Referencias}

Bourdieu, P. (2000). La dominación masculina. España: Editorial ANAGRAMA

Habermas, J. (1998). Facticidad y validez. Sobre el derecho y el estado de derecho en términos de teoría del discurso. Editorial Trotta.

Lamas, M. (1996). Problemas sociales causados por el género. México: Ibero.

Lamas, M. (2000). Diferencias de sexo, género y diferencia sexual. Cuicuilco, 7(18), 1-24.

Pérez, Y. (2013). Participación comunitaria en la vialidad rural en el marco de la Ley 8114 de Simplificación y Eficiencia Tributaria, El caso del cantón de Naranjo. Tesis de Maestría.

Proyecto MOPT/GTZ. (2001). Guía Práctica para la aplicación del Modelo de Conservación Vial Participativa. Costa Rica: GTZ

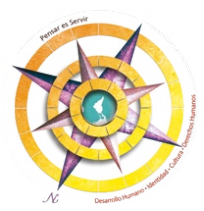

"Los caminos no tienen sexo ni género" 119

Una propuesta para la incorporación de mujeres en el espacio público comunitario en el marco de la infraestructura vial cantonal, Naranjo, Costa Rica Yolanda Pérez-Carrillo 
